\title{
Robinsoniella peoriensis gen. nov., sp. nov., isolated from a swine-manure storage pit and a human clinical source
}

\author{
Correspondence \\ Paul A. Lawson \\ paul.lawson@ou.edu
}

\author{
Michael A. Cotta, ${ }^{1}$ Terence R. Whitehead, ${ }^{1}$ Enevold Falsen, ${ }^{2}$ \\ Edward Moore ${ }^{2}$ and Paul A. Lawson ${ }^{3}$ \\ ${ }^{1}$ Fermentation Biotechnology Research Unit, National Center for Agricultural Utilization Research, \\ USDA, Agricultural Research Service, 1815 N. University Street, Peoria, IL 61604, USA \\ ${ }^{2}$ Culture Collection, Department of Clinical Bacteriology, University of Göteborg, S-41346 \\ Göteborg, Sweden \\ ${ }^{3}$ Department of Botany and Microbiology, University of Oklahoma, OK 73019, USA
}

The intensification of modern livestock-production systems and the concentration of greater numbers of animals into fewer operations has led to the inevitable concentration of waste products (generated by these facilities) into increasingly smaller locations. The methods used to manage liquid swine manure in relation to such farming practices are lagoon treatment or deep-pit storage. A consequence of these storage processes is the production of a variety of odorous chemicals, including ammonia, organic acids and alcohols and sulphides; these cause a nuisance and not only can pose health problems for both the animals and the staff, but may also lead to contamination of local water supplies as a result of runoff of liquids. The production of these chemicals is the result of microbiological activity and, consequently, the underlying microbiology of these processes is the focus of ongoing research on swine-manure management. During a continuing study into the microflora of lagoons and manure pits, five strains of a Gram-positive-staining,

The GenBank/EMBL/DDBJ accession number for the $16 \mathrm{~S}$ rRNA gene sequence of strain PPC $31^{\top}$ is AF445285.

Details of the polar lipids of strain PPC $31^{\top}$ and an unrooted, maximumparsimony phylogenetic tree for strain $\mathrm{PPC} 31^{\top}$ and members of the family Lachnospiraceae are available as supplementary figures with the online version of this paper. strictly anaerobic, spore-forming, rod-shaped organism were isolated (Cotta et al., 2003). When a representative strain was deposited with the Culture Collection of the University of Göteborg (Göteborg, Sweden) for further characterization, it was found to be biochemically and phylogenetically related to a clinical strain recovered from a human source. On the basis of phenotypic and phylogenetic evidence, it is proposed that the unknown bacterium originating from swine-waste lagoons and human blood represents a novel genus and species.

Five isolates, designated PPC $31^{\mathrm{T}}$, PC37, PC47, PC50 and PC108, were recovered from both fresh swine manure and manure storage pits. During the course of this investigation, an additional strain deposited with the Culture Collection of the University of Göteborg showed a very similar biochemical profile and was designated CCUG 52336. Strain CCUG 52336 was isolated from a deep wound on the heel of a 79-year-old female and was cultured anaerobically at $37{ }^{\circ} \mathrm{C}$ on chocolate agar. The unidentified swine-manure organisms were cultured on basic media containing macrominerals, microminerals, buffers, reducing agents and other components, as in the routine growth medium described by Hespell et al. (1987), with $0.2 \%$ carbohydrate added as a carbon source. All of the media used in these experiments were prepared 
anaerobically using the method of Hungate, as modified by Bryant (1972). Fermentation end products were determined using GC and HPLC methods. GC was performed on a Hewlett Packard model 5890A gas chromatograph with an HP Innowax capillary column $(30 \mathrm{~m} \times 0.32 \mathrm{~mm}$, $0.5 \mu \mathrm{m}$ film thickness; Agilent Technologies) (Miller, 2001). HPLC of organic acids was performed using a Bio-Rad Aminex HPX-87H column at $65{ }^{\circ} \mathrm{C}$ with $5 \mathrm{mM}$ $\mathrm{H}_{2} \mathrm{SO}_{4}$ as the solvent. Peaks were detected with a differential refractometer (model 410; Waters) and identified by means of comparison with the retention times of authentic standards (Cotta et al., 2003).

The unknown organism recovered from the manure storage pit and a human source consisted of Grampositive, ovoid to short rod-shaped cells. Cells appeared to be non-motile in the $0.4 \%$ agar tube test (Bailey \& Scott, 1974). Microscopic observation revealed that spores were located subterminally; cells were not swollen. Incubation at $37{ }^{\circ} \mathrm{C}$ for 2 days under anaerobic conditions or in air plus $5 \% \mathrm{CO}_{2}$ on tryptic soy agar supplemented with $5 \%$ defibrinated horse blood produced colonies that were 0.5 $1.5 \mathrm{~mm}$ in diameter and did not demonstrate haemolytic activity. The organism was found to utilize amygdalin, arabinose, cellobiose, fructose, glucose, maltose, lactose, raffinose, starch, trehalose, xylan and xylose but not cellulose. The strains were biochemically characterized by using the API Rapid ID 32AN and API ZYM systems according to the manufacturer's instructions (bioMérieux). With the API Rapid ID 32AN system, positive reactions were obtained for $N$-acetyl- $\beta$-glucosaminidase, $\alpha$-arabinosidase, $\alpha$-galactosidase, $\beta$-galactosidase, $\alpha$-glucosidase, $\beta$ glucosidase, $\beta$-glucuronidase and $\alpha$-fucosidase. Negative reactions were obtained for alanine arylamidase, alkaline phosphatase, arginine arylamidase, arginine dihydrolase, glutamic acid decarboxylase, glutamyl glutamic acid arylamidase, glycine arylamidase, histidine arylamidase, leucine arylamidase, leucyl glycine arylamidase, mannose, phenylalanine arylamidase, proline arylamidase, pyroglutamic acid arylamidase, raffinose, serine arylamidase, tyrosine arylamidase and urease. With the API ZYM system, positive reactions were obtained for $N$-acetyl $\beta$ glucosaminidase (weak), acid phosphatase, esterase (C4) (weak), esterase lipase (C8) (weak), $\alpha$-glucosidase, $\beta$ glucosidase (weak), $\beta$-galactosidase, $\beta$-glucuronidase and naphthol-AS-BI-phosphohydrolase. All other reactions in the API ZYM test system were negative. Nitrate was not reduced and indole was not produced.

Long-chain cellular fatty acids were extracted and analysed by gas chromatography (MIDI Sherlock) as described previously (Kämpfer \& Kroppenstedt, 1996). The swine faecal strains and the clinical strain gave very similar longchain cellular fatty acid profiles that consisted of a complex mixtures of fatty acids, dimethylacetals and aldehydes and were found to consist primarily of the unbranched saturated and unsaturated types; iso- and anteiso-methylbranched forms were present only in very small amounts. The quantitative fatty acid data for $\mathrm{PPC} 31^{\mathrm{T}}$ were as follows:
$\mathrm{C}_{13: 1}(2.7 \%), \mathrm{C}_{13: 0} 3-\mathrm{OH}(8.2 \%), \mathrm{C}_{14: 0}(11.4 \%), \mathrm{C}_{16: 0}$ $(31.0 \%), \mathrm{C}_{16: 0} \operatorname{ALD}(1.3 \%), \mathrm{C}_{16: 1} \omega 7 c(1.9 \%), \mathrm{C}_{16: 1} \omega 7 c$ $(10.0 \%)$, iso- $\mathrm{C}_{17: 1}(7.1 \%), \mathrm{C}_{17: 1} \omega 8 c(2.3 \%), \mathrm{C}_{18: 1} \omega 7 c$ $(3.8 \%), \quad \mathrm{C}_{18: 0} \quad(1.3 \%), \quad \mathrm{C}_{18: 1} \omega 9 \mathrm{c} \quad(1.4 \%), \quad \mathrm{C}_{18: 1} \omega 7 c$ (12.2\%), $\mathrm{C}_{19: 0} \omega 8 \mathrm{c}$ cyclo $(2.1 \%)$ and $\mathrm{C}_{19: 0}(2.4 \%)$.

Analyses of cell-wall sugars and peptidoglycan, respiratory quinones and polar lipids in strain PPC $31^{\mathrm{T}}$ were carried out by the Identification Service of the Deutsche Sammlung von Mikroorganismen und Zellkulturen (DSMZ; Braunschweig, Germany) and Dr B. J. Tindall (DSMZ). The whole-cell-wall sugars of strain PPC $31^{\mathrm{T}}$ consisted of galactose, glucose, rhamnose and large amounts of ribose. Analysis of cell-wall murein hydrolysates from the isolate revealed the presence of mesodiaminopimelic acid as the diagnostic diamino acid. No respiratory quinones were detected. The polar lipid profile comprised an aminophosphoglycolipid, an aminophospholipid, diphosphatidylglycerol, glycolipids, phospholipids, phosphatidylethanolamine and phosphatidylglycerol (Supplementary Fig. S1, available in IJSEM Online).

Determination of the DNA G $+C$ content was carried out by means of thermal denaturation of chromosomal DNA, using a Beckman spectrophotometer (model DU 640) equipped with a high-performance temperature controller and $T_{\mathrm{m}}$ analysis software (Johnson, 1994). The $\mathrm{G}+\mathrm{C}$ content of the DNA of strain PPC $31^{\mathrm{T}}$ was determined to be $48.7 \mathrm{~mol} \%$. 16S rRNA gene fragments were generated by using a PCR with universal primers pA (positions 8-28, Escherichia coli numbering) and $\mathrm{pH}^{\star}(1542-1522)$. The amplified product was purified using a QIAquick PCR purification kit (Qiagen) and sequenced directly using primers to conserved regions of the rRNA gene. Sequencing was performed using a PRISM Taq DyeDeoxy Terminator cycle sequencing kit (Applied Biosystems) and an automatic DNA sequencer (model 373A; Applied Biosystems). The closest known relatives of the novel isolate were determined by performing database searches using the program FASTA (Pearson \& Lipman, 1985). These sequences and those of other known related strains were retrieved from GenBank and aligned with the newly determined sequence by using the program ARB (Ludwig et al., 2004). Phylogenetic analysis was performed on 1320 shared nucleotides by using the neighbour-joining and maximum-parsimony algorithms; the stability of the groupings was estimated by means of bootstrap analysis (1000 replications) using the same programs. The six isolates were closely related to each other genetically (99.4$100 \% 16 \mathrm{~S}$ rRNA gene sequence similarity). Sequence searches of the GenBank and Ribosomal Database Project libraries revealed that the unknown organism was a member of the phylum Firmicutes within the family Lachnospiraceae. It was evident from treeing analysis that the unknown bacterium represented a previously unknown lineage within the clostridial rRNA cluster XIVa subgroup. Pairwise comparisons revealed approximately $5.0 \%$ sequence divergence between the unknown bacterium and the type strains of the closest species with validly 
published names, i.e. Ruminoccus luti and Ruminoccus obeum, based on almost-complete sequences of the $16 \mathrm{~S}$ rRNA gene (1400 nt). A phylogenetic tree, constructed using the neighbour-joining method, depicting the phylogenetic affinity of the novel bacterium (exemplified by strain $P P C 31^{\mathrm{T}}$ ) is shown in Fig. 1. All of the major branching nodes were confirmed by maximum parsimony (Supplementary Fig. S2). There was some rearrangement of the branching order within the ruminococcal cluster because of the similar sequence divergence values between some of these species, but the placement of the novel organism at the base of this cluster was confirmed.

There is no precise correlation between 16S rRNA gene sequence divergence and species delineation, but it is generally recognized that divergence values of $3 \%$ or more are significant (Stackebrandt \& Goebel, 1994). Although tree topology and sequence divergence values $(>5 \%)$ show that the unidentified ovoid organism warrants classification as a novel species, its generic assignment is somewhat problematic.

The Clostridium coccoides group (clostridial rRNA cluster XIVa; Collins et al., 1994) contains a diverse assortment of organisms within the family Lachnospiraceae and includes the genera Acetitomaculum, Anaerostipes, Bryantella, Butyrivibrio, Catonella, Coprococcus, Dorea, Hespellia, Johnsonella, Lachnospira, Lachnobacterium, Moryella, Oribacterium, Parasporobacterium, Pseudobutyrivibrio, Roseburia, Shuttleworthia, Sporobacterium and Syntrophococcus in addition to many misclassified clostridial species. However, the novel organism forms a distinct lineage within this large grouping and does not display a particularly close affinity with any of the aforementioned taxa. In this diverse group of organisms it is clear that no single organism possesses a completely unique biochemical profile; indeed, data for classical taxonomic markers such as polar lipids and cell-wall murein types are missing from both well-established and newly described genus descriptions. Furthermore, the most basic of the discriminatory tests - the Gram stain - is increasingly being called into question (Shah et al., 1997), with a number of Gramnegative taxa (viz. Catonella morbi, Johnsonella ignava) being placed phylogenetically within the Clostridium coccoides (rRNA cluster XIVa) group of organisms. Thus, accurate identification, especially at the laboratory bench, is becoming ever more reliant on rapid molecular genetic techniques, in particular 16S rRNA gene sequence comparisons. Although these high-throughput methodologies were previously performed only in specialized facilities, they are becoming increasingly automated and are therefore cheaper, making them more accessible for routine laboratory work and the services offered by commercial facilities. This allows accurate identification of hitherto unknown taxa in a turnaround time of a matter of hours or days. Such changes are reflected in the latest edition of the Bergey's Manual of Systematic Bacteriology, the format of which now involves a phylogenetic basis for the classification of micro-organisms. We do not advocate that traditional or classical tests be abandoned or their importance eroded; indeed, these methods must be maintained to a high standard. However, taxonomy should be a practical field in which polyphasic techniques are brought together to facilitate the identification of the everincreasing number of novel taxa. It is clear from phylogenetic molecular profiling studies of the gastrointestinal tracts of humans and animals that, in addition to

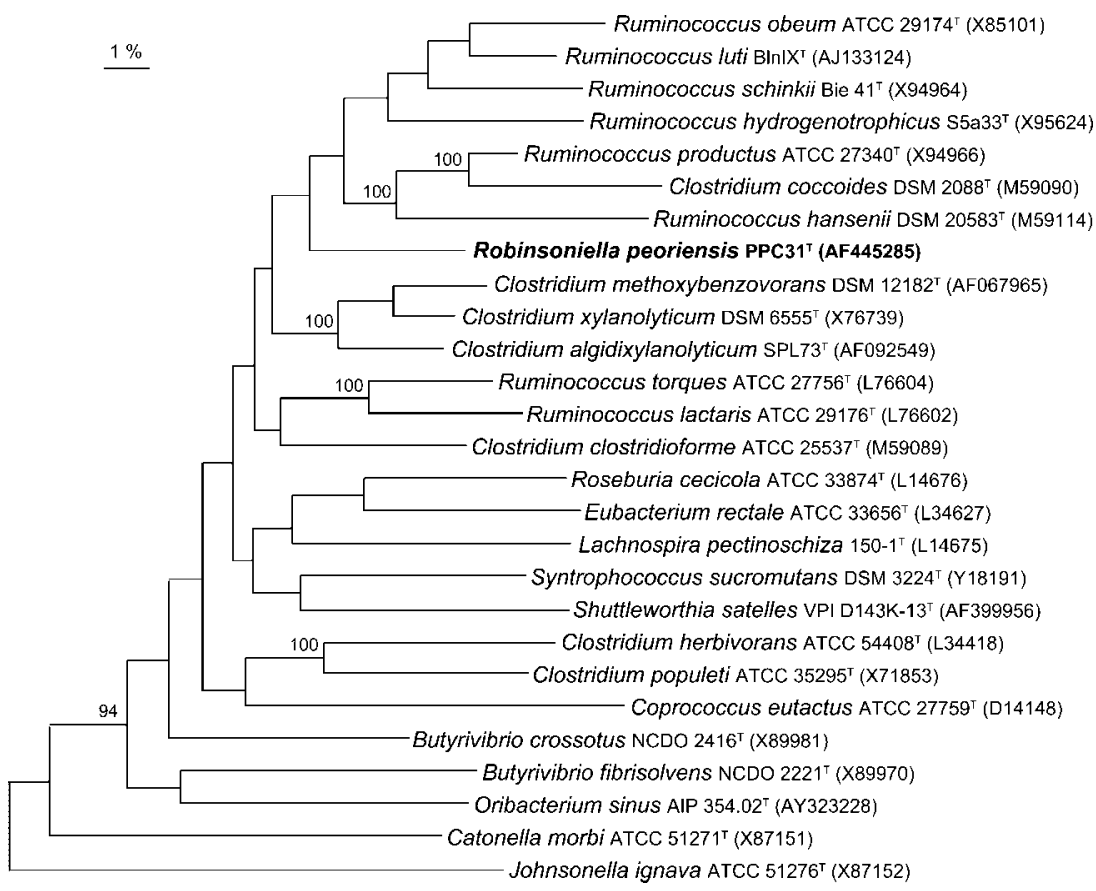

Fig. 1. Unrooted tree showing the phylogenetic relationships of strain $\mathrm{PPC} 31^{\top}$ and some other organisms within the family Lachnospiraceae. The tree, constructed using the neighbour-joining method, was based on a comparison of approx. $1320 \mathrm{nt}$. Bootstrap percentages (based on 1000 replications) are given at branching points; only values $\geqslant 90 \%$ are shown. Bar, $1 \%$ sequence divergence. 
the large family of organisms contained in rRNA clostridial cluster XIVa, many taxa remain to be cultured, isolated and characterized (Barcenilla et al., 2000; Hold et al., 2002; Leser et al., 2002; Namsolleck et al., 2004; Suau et al., 1999). The growing reality is that, with the plethora of taxa now being described, genus descriptions will cease to be sufficiently unique to allow accurate identifications to be based solely on phenotypic criteria. An examination of the literature demonstrates that many descriptions of novel genera are little more than 'anaerobic, Gram-positive rods that ferment a number of carbohydrates' and, in addition, may be represented by a single strain.

The novel organism described here represents a unique line of descent and is phylogenetically located at the base of a cluster of organisms consisting of a number of misclassified ruminococci and indeed shares some morphological and biochemical traits with these species, although this relationship is not supported by a significant bootstrap value. However, the novel organism produces spores and therefore should not be included within this group (although it is pertinent to note that Clostridium coccoides, a species that is not associated with humans, does produce spores). Also included within this supra-generic grouping are a number of misclassified clostridial (and many other) taxa, as stated previously. On the basis of the recommendation of Collins et al. (1994), true clostridia should be restricted to those species contained in rRNA cluster 1; similarly, the unknown isolates are incompatible with eubacteria, as they do not produce butyrate as a major fermentation product. They are distinguishable from members of the genus Lachnospira by the absence of curved cellular shapes and can be differentiated from Roseburia and Butyrivibrio on the basis of the end products of glucose metabolism (i.e. no butyric acid production) and by the absence of motility. As the phenotypic and phylogenetic data demonstrate its separateness from other members of the Clostridium coccoides group, we consider that the unidentified organism merits classification within a novel genus, for which the name Robinsoniella gen. nov. is proposed. In addition to its unique 16S rRNA gene sequence, the novel organism can be differentiated from its closest phylogenetic relatives by means of biochemical and chemotaxonomic markers, such as the presence of a diagnostic murein type based on meso-diaminopimelic acid and the presence of a complex mixture of polar lipids. The novel organism can be distinguished from Clostridium coccoides and misclassified ruminococci by using the tests shown in Table 1 and can be differentiated from other closely related organisms within the clostridial rRNA XIVa cluster of organisms by using the tests shown in Table 2 .

\section{Description of Robinsoniella gen. nov.}

Robinsoniella (Ro.bin.so.ni.el'la. N.L. fem. dim. n. Robinsoniella in honour of Isadore M. Robinson, in recognition of his many contributions to swine microbiology).

Cells are Gram-positive, spore-forming, ovoid to short rods found as single cells or in pairs. Anaerobic. The cellwall murein is based on meso-diaminopimelic acid as the diagnostic diamino acid. No respiratory quinones are detected. Polar lipids include an aminophosphoglycolipid, an aminophospholipid, diphosphatidylglycerol, glycolipids, phospholipids, phosphatidylethanolamine and phosphatidylglycerol. The major long-chain cellular fatty acid profile consists of a complex mixture of straight-chain saturated, monounsaturated and iso-methyl-branched acids. The

Table 1. Characteristics that can be used to distinguish Robinsoniella peoriensis gen. nov., sp. nov. from closely related ovoid organisms

Species: 1, Robinsoniella peoriensis; 2, Clostridium coccoides; 3, Ruminococcus gnavus; 4, Ruminococcus hydrogenotrophicus; 5, Ruminococcus hensenii; 6, Ruminococcus lactaris; 7, Ruminococcus luti; 8, Ruminococcus obeum; 9, Ruminococcus productus; 10, Ruminococcus schinkii; 11, Ruminococcus torques. Data are from Ezaki et al. (2006), Simmering et al. (2002) and this study. +, Positive; w, weak reaction; -, negative; d, different among strains; ND, no data.

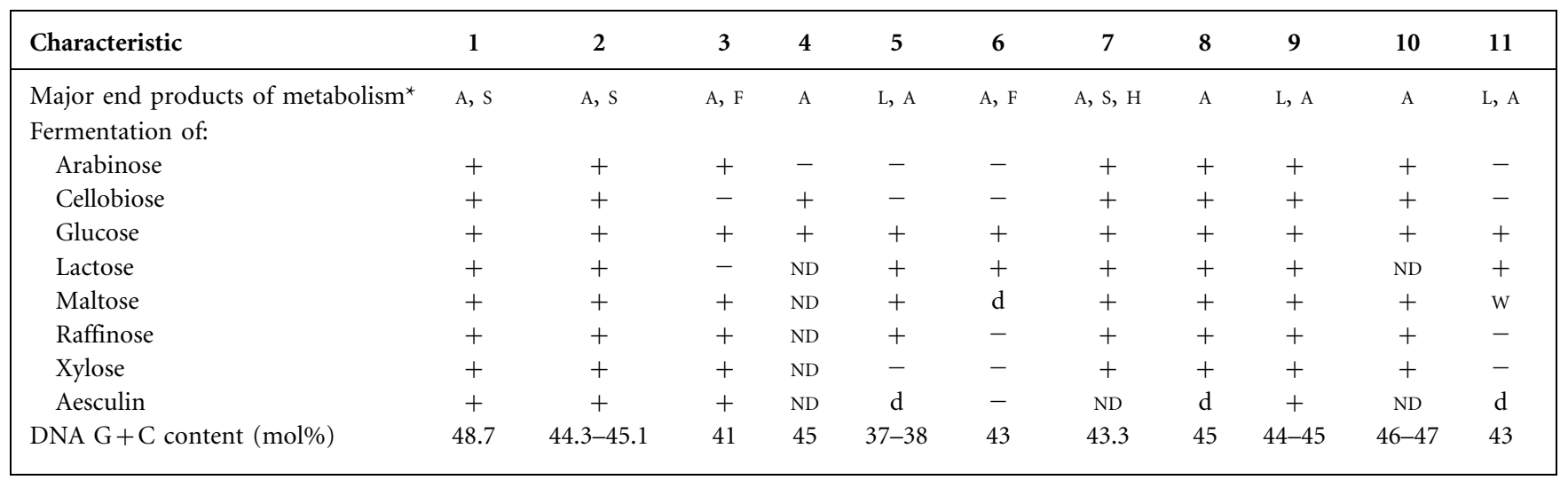

${ }^{*}$ A, Acetate; F, formate; H, hydrogen; L, lactate; s, succinate. 


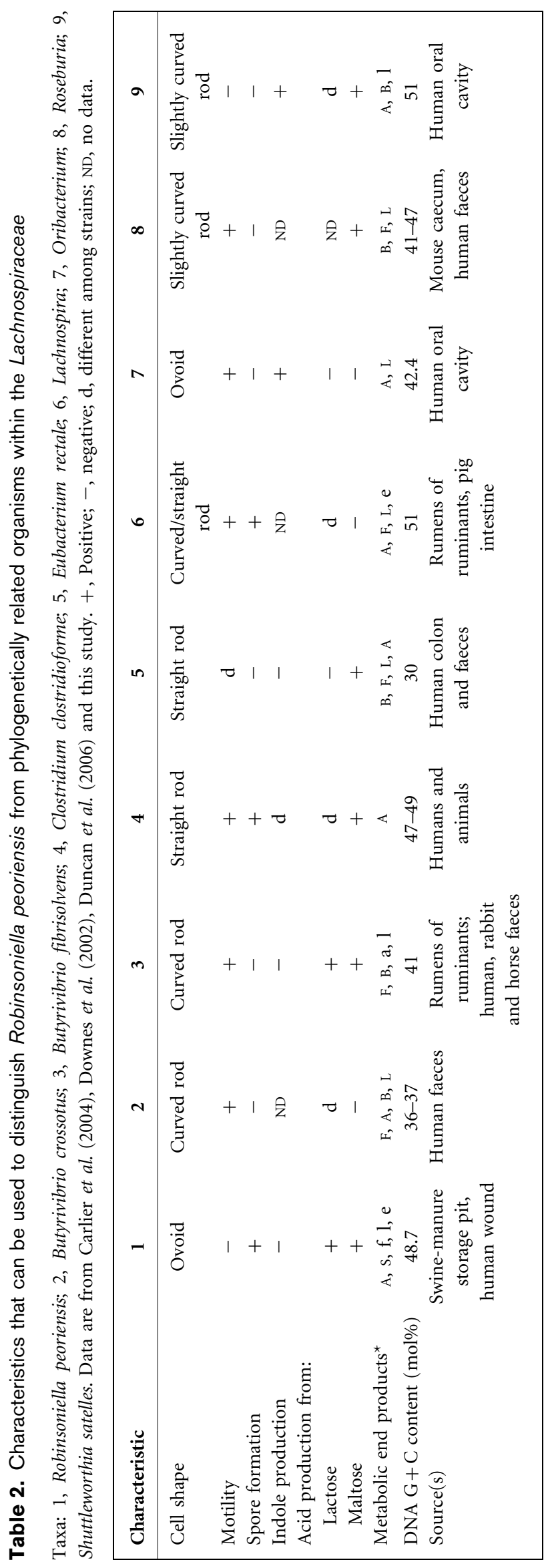

major end products of metabolism include acetate and succinate, but no butyrate is detected. Growth is observed with glucose in addition to a range of carbohydrates. The DNA G $+\mathrm{C}$ content is approximately $49 \mathrm{~mol} \%$. The type species of the genus is Robinsoniella peoriensis. Comparative analysis of the 16S rRNA gene sequence shows that the genus Robinsoniella represents a separate line of descent within the family Lachnospiraceae.

\section{Description of Robinsoniella peoriensis sp. nov.}

Robinsoniella peoriensis (pe.o'ri.en.sis. N.L. fem. adj. peoriensis pertaining to Peoria, a city in Illinois, USA, from where the type strain was isolated).

Has the following characteristics in addition to those described for the genus. Produces subterminal spores that do not cause the cells to swell. With the API Rapid ID $32 \mathrm{AN}$ and API ZYM systems, positive reactions are obtained for $N$-acetyl- $\beta$-glucosaminidase, $\alpha$-arabinosidase, $\alpha$-galactosidase, $\beta$-galactosidase, $\alpha$-glucosidase, $\beta$-glucosidase, $\beta$-glucuronidase, $\alpha$-fucosidase, acid phosphatase, esterase (C4) (weak), esterase lipase (C8) (weak) and naphthol-AS-BI-phosphohydrolase. Nitrate is not reduced and indole is not produced. The cell-wall sugars consist of galactose, glucose, rhamnose and large amounts of ribose. The major long-chain fatty acids are $\mathrm{C}_{13: 0} 3-\mathrm{OH}, \mathrm{C}_{14: 0}$, $\mathrm{C}_{16: 0}, \mathrm{C}_{16: 1} \omega 7 c$, iso- $\mathrm{C}_{17: 1}$ and $\mathrm{C}_{18: 1} \omega 7 c$. The DNA G+C content of the type strain is $48.7 \mathrm{~mol} \%$.

The type strain, PPC $31^{\mathrm{T}}\left(=\mathrm{CCUG} 48729^{\mathrm{T}}=\right.$ NRRL B$23985^{\mathrm{T}}$ ), was isolated from a swine-manure storage pit. Additional strains PC37 (=NRRL B-51275), PC47 (=NRRL B-51276), PC50 (=NRRL B-51277) and PC108 (=NRRL B-51278) were recovered from both fresh swine manure and manure storage pits. Strain CCUG 52336 (=NRRL B-51274) was isolated in Sweden from a deep human wound. Habitat unknown.

\section{Acknowledgements}

The authors wish to acknowledge the excellent technical assistance of Rhonda Zeltwanger. We also thank Hans Trüper and Jean Euzéby for assistance with the genus and species epithets.

\section{References}

Bailey, W. R. \& Scott, E. G. (1974). In Diagnostic Microbiology, 4th edn, pp. 376-377. St. Louis, MO: C. V. Mosby.

Barcenilla, A., Pryde, S. E., Martin, J. C., Duncan, S. H., Stewart, C. S., Henderson, C. \& Flint, H. J. (2000). Phylogenetic relationships of butyrate-producing bacteria from the human gut. Appl Environ Microbiol 66, 1654-1661.

Bryant, M. P. (1972). Commentary on the Hungate technique for culture of anaerobic bacteria. Am J Clin Nutr 25, 1324-1328.

Carlier, J.-P., K’Ouas, G., Bonne, l., Lozniewski, A. \& Mory, F. (2004). Oribacterium sinus gen. nov., sp. nov., within the family 'Lachnospiraceae' (phylum Firmicutes). Int J Syst Evol Microbiol 54, 1611-1615. 
Collins, M. D., Lawson, P. A., Willems, A., Cordoba, J. J., FernandezGarayzabal, J., Garcia, P., Cai, J., Hippe, H. \& Farrow, J. A. E. (1994). The phylogeny of the genus Clostridium: proposal of five new genera and eleven new species combinations. Int J Syst Bacteriol 44, 812-826.

Cotta, M. A., Whitehead, T. R. \& Zeltwanger, R. L. (2003). Isolation, characterization and comparison of bacteria from swine faeces and manure storage pits. Environ Microbiol 5, 737-745.

Downes, J., Munson, M. A., Radford, D. R., Spratt, D. A. \& Wade, W. G. (2002). Shuttleworthia satelles gen. nov., sp. nov., isolated from the human oral cavity. Int J Syst Evol Microbiol 52, 1469-1475.

Duncan, S. H., Aminov, R. I., Scott, K. P., Louis, P., Stanton, T. B. \& Flint, H. J. (2006). Proposal of Roseburia faecis sp. nov., Roseburia hominis sp. nov. and Roseburia inulinivorans sp. nov., based on isolates from human faeces. Int J Syst Evol Microbiol 56, 2437-2441.

Ezaki, T., Li, N. \& Kawamura, Y. (2006). The anaerobic gram-positive cocci. In The Prokaryotes, 3rd edn, vol. 6, pp. 795-808. Edited by M. Dworkin, S. Falkow, E. Rosenberg, K. H. Schleifer \& E. Stackebrandt. New York: Springer.

Hespell, R. B., Wolf, R. \& Bothast, R. J. (1987). Fermentation of xylans by Butyrivibrio fibrisolvens and other ruminal bacteria. Appl Environ Microbiol 53, 2849-2853.

Hold, G. L., Pryde, S. E., Russell, V. J., Furrie, E. \& Flint, H. J. (2002). Assessment of microbial diversity in human colonic samples by $16 \mathrm{~S}$ rDNA sequence analysis. FEMS Microbiol Ecol 39, 33-39.

Johnson, J. L. (1994). Similarity analysis of DNAs. In Methods for General and Molecular Bacteriology, pp. 656-682. Edited by P. Gerhardt, R. G. E. Murray, W. A. Wood \& N. R. Krieg. Washington, DC: American Society for Microbiology.

Kämpfer, P. \& Kroppenstedt, R. M. (1996). Numerical analysis of fatty acid patterns of coryneform bacteria and related taxa. Can J Microbiol 42, 989-1005.
Leser, T. D., Amenuvor, J. Z., Jensen, T. K., Lindecrona, R. H., Boye, M. \& Moller, K. (2002). Culture-independent analysis of gut bacteria: the pig gastrointestinal tract microbiota revisited. Appl Environ Microbiol 68, 673-690.

Ludwig, W., Strunk, O., Westram, R., Richter, L., Meier, H., Yadhukumar, Buchner, A., Lai, T., Steppi, S. \& other authors (2004). ARB: a software environment for sequence data. Nucleic Acids Res 32, 1363-1371.

Miller, D. N. (2001). Accumulation and composition of odorous compounds in feedlot soils under aerobic, fermentative, anaerobic respiratory conditions. J Anim Sci 79, 2503-2512.

Namsolleck, P., Thiel, R., Lawson, P., Holmstrøm, K., Rajilic, M., Vaughan, E. E., Rigottier-Gois, L., Collins, M. D., de Vos, W. M. \& Blaut, M. (2004). Molecular methods for the analysis of gut microbiota. Microb Ecol Health Dis 16, 71-85.

Pearson, W. R. \& Lipman, D. J. (1985). Rapid and sensitive protein similarity searches. Science 227, 1435-1441.

Shah, H. N., Gharbia, S. E. \& Collins, M. D. (1997). The gram-stain, a declining synapomorphy in an emerging evolutionary tree. Rev Med Microbiol 8, 103-110.

Simmering, R., Taras, D., Schwiertz, A., Le Blay, G., Gruhl, B., Lawson, P. A., Collins, M. D. \& Blaut, M. (2002). Ruminococcus luti sp. nov., isolated from a human faecal sample. Syst Appl Microbiol 25, 189-193.

Stackebrandt, E. \& Goebel, B. M. (1994). Taxonomic note: a place for DNA-DNA reassociation and 16S rDNA sequence analysis in the present species definition in bacteriology. Int J Syst Bacteriol 44, 846849.

Suau, A., Bonnet, R., Sutren, M., Godon, J.-J., Gibson, G. R., Collins, M. D. \& Doré, J. (1999). Direct analysis of genes encoding $16 \mathrm{~S}$ rRNA from complex communities reveals many novel molecular species within the human gut. Appl Environ Microbiol 65, 4799-4807. 